\title{
Optical coherence tomography-measured retinal nerve fiber layer thickness values compensated with a multivariate model and discrimination between stable and progressing glaucoma suspects
}

\author{
Hemma Resch ${ }^{1} \cdot$ Florian Schwarzhans $^{2} \cdot$ Florian Frommlet $^{3} \cdot$ Anton Hommer $^{4} \cdot$ Philipp Fuchs $^{1} \cdot$ Clemens Vass $^{1}$ (D)
}

Received: 29 January 2021 / Revised: 9 July 2021 / Accepted: 16 July 2021 / Published online: 5 August 2021

(C) The Author(s) 2021, corrected publication 2021

\begin{abstract}
Purpose Our previously introduced multivariate model, compensating for intersubject variability, was applied to circumpapillary retinal nerve fiber layer (RNFL) values measured with optical coherence tomography in glaucoma suspects with or without prior progressive optic disc (OD) change in a series of confocal scanning laser tomography (CSLT, HRT III) measurements.

Methods In this prospective study, OD change during CSLT follow-up was determined with strict, moderate, and liberal criteria of the topographic change analysis (TCA). Model compensation (MC) as well as age compensation (AC) was applied to RNFL sectors (RNFLMC vs. RNFLAC). Diagnostic performance of RNFLMC vs. RNFLAC was tested with an area under the receiver operating characteristic (AUROC) and was compared between methods.

Results Forty-two glaucoma suspects were included. Patients without prior progressive OD change during the CSLT followup (= stable) had thicker RNFL thickness values in most areas and for all progression criteria. RNFLMC AUROC for the global RNFL (0.719) and the inferior quadrant (0.711) performed significantly better compared with RNFLAC AUROC (0.594 and 0.631) to discriminate between stable and progressive glaucoma suspects as defined by the moderate criteria of CSLT progression analysis $(p=0.028 ; p=0.024)$.

Conclusion MC showed a slight but significant improvement in detection of subjects with prior progressive OD change among a group of glaucoma suspects, when compared to AC, which is the compensation method commonly used during OCT data evaluation in daily routine. Further studies are warranted to validate the present results.
\end{abstract}

Keywords Optic nerve · Glaucoma $\cdot$ Imaging $\cdot$ HRT/CSLT $\cdot$ OCT

Clemens Vass

clemens.vass@meduniwien.ac.at

1 Department of Ophthalmology \& Optometry, Medical University Vienna, General Hospital, Waehringer Guertel 18-20, A-1090 Vienna, Austria

2 Section for Medical Information Management and Imaging, Center for Medical Statistics Informatics and Intelligent Systems, Medical University Vienna, Vienna, Austria

3 Section for Medical Statistics, Center for Medical Statistics Informatics and Intelligent Systems, Medical University Vienna, Vienna, Austria

4 Hommer Ophthalmology Institute, Albertgasse 39, Vienna, Austria 


\section{Key messages}

- A multivariate model including eight individual factors to compensate for intersubject variability was applied to circumpapillary RNFL values measured with OCT (Cirrus $\left.{ }^{\circledR}\right)$ in glaucoma suspects with or without prior progressive optic disc change in a series of confocal scanning laser tomography (CSLT) (HRT®III) measurements.

- Glaucoma suspect patients with prior progressive OD change during the CSLT follow-up had thinner RNFL thickness values in most areas and for all progression criteria.

- The model compensation of RNFL thickness measurements compared to age compensation performed better in detection of progressing cases among glaucoma suspects.

- Including a set of individual factors in the interpretation of peripapillary RNFL measurement with OCT in glaucoma management in the daily routine might improve the diagnosis of glaucoma cases among glaucoma suspects.

\section{Introduction}

Glaucoma is a progressive optic neuropathy characterized by the irreversible loss of retinal ganglion cells and their axons, leading to visual field defects [1]. An accurate and early diagnosis of this chronic disease is crucial, as no cure is established.

Currently, early diagnosis of glaucoma, besides functional tests, is performed based on imaging techniques such as optical coherence tomography (OCT) [2]. This is done by measuring the circumpapillary retinal nerve fiber layer (RNFL) thickness and optic disc (OD) parameters and comparing the measurement results with a normative database. Despite a high reproducibility [3, 4] of RNFL thickness measurements, intersubject variance [5] due to the influence of numerous individual factors hampers the interpretation and use of imaging techniques, especially in the early diagnosis of glaucoma. Individual biomarkers, such as sex, age, and ethnicity [6-9], are typically taken into account by the normative databases. However, other factors are not, including optic disc and fovea parameters, retinal vessel position, axial length, and refractive error, although they show an association with circumpapillary RNFL thickness and distribution [10-19].

In a previous publication, which is in line with previous research, we could show a significant correlation of retinal blood vessel position and peripapillary RNFL thickness distribution [20-22]. To enable a more comprehensive analysis of this factor, we recently have developed the parameter of circumpapillary retinal vessel density (RVD) - a function dependent on thickness and distribution of the major circumpapillary retinal blood vessels [23]. We have shown that interindividual variance may be reduced, on average, by $11 \%$ and up to $20 \%$ when taking RVD into account.

In a further study [24], this parameter, together with seven other anatomical features (corresponding to OD and fovea descriptors, as well as age and refractive error), were included in a multivariate model. The sample used to develop the multivariate model consisted of 101 healthy subjects and the independent sample used to validate the model consisted of a different set of 101 healthy subjects. The model achieved a reduction of the coefficient of variation of $18 \%$ on average (up to $29 \%$ ), when tested in the independent validation sample. This work provides strong evidence of the major impact that anatomical factors, especially the distribution of the retinal vessels, have on the RNFL thickness profile in healthy volunteers. The reduction of the coefficient of variance demonstrates that intersubject variance in the RNFL thickness profile of healthy subjects can be decreased, which may improve the imaging support of glaucoma diagnosis.

In the present study, we have included glaucoma suspects, an interesting patient group, as the differentiation between normal and suspect-looking optic discs in early glaucoma is very much complicated especially due to interindividual differences. Our previously introduced model, compensating for intersubject variability, was applied to circumpapillary RNFL values measured with OCT (Cirrus ${ }^{\circledR}$ ) in glaucoma suspects with or without prior progressive OD change in a series of at least 4 confocal scanning laser tomography (CSLT) (HRT®III) measurements. The aim of the present study was to investigate whether glaucoma suspect patients with or without prior OD change are behaving differently in RNFL measurement with OCT and if reducing interindividual variability of RNFL measurements through using a multivariate compensation method might improve detection of glaucoma among glaucoma suspects. 


\section{Materials and methods}

\section{Subjects}

This prospective study was performed in collaboration with the Department of Ophthalmology and Optometry and the Section for Medical Statistics, Center for Medical Statistics Informatics and Intelligent Systems, Medical University Vienna, Vienna, Austria, for data analysis.

\section{Inclusion and exclusion criteria}

Forty-two glaucoma suspects were included. They were recruited from an ophthalmology institute, where follow-up examinations with the CSLT (HRT) during their glaucoma routine checks were done at least once a year prior to this study.

Inclusion criteria for glaucoma suspects were suspect appearance of the OD of at least one eye (either large excavation $>0.5$, or asymmetry of excavation $>0.2$, or localized rim loss, or failure of ISNT rule, or baring of circumlinear vessels) and reproducible normal visual fields (VF) in standard automated perimetry (SAP). Progressive OD change during a series of 3 of at least 4 consecutive CSLT follow-up measurements was determined with strict, moderate, and liberal criteria of the topographic change analysis (TCA) [25].

Patients with or without prior progressive OD change were divided into 2 groups, progressive or stable. This was done for all three TCA criteria.

We excluded all subjects with any evidence of other ocular pathology, history of ocular trauma or intraocular surgery, ocular inflammation or infection within the last 3 months, astigmatism more than +2.0 diopters, and ametropia of more than \pm 5.0 diopters. In addition to these criteria, only optic disc examinations acquired with Fourier Domain OCT (FD-OCT, Cirrus ${ }^{\circledR}$ Carl Zeiss Meditec Inc.) with a quality index higher than 5 were included (quality index was ranging from 0 to 10 ), as image quality may have an impact on RNFL thickness measurements [26]. Moreover, scans with movement artifacts within the measurement circle were excluded.

\section{Experimental paradigm}

Initially, a complete ophthalmological examination was performed, including medical history, best-corrected Snellen visual acuity, slit-lamp examination, fundoscopy, measurement of IOP by Goldmann applanation tonometry, and VF examination.

Subjects eligible for participating in the study according to the inclusion/exclusion criteria were included. If both eyes were includable, one eye was selected randomly.
The study was performed at the Department of Ophthalmology and Optometry of the Medical University of Vienna.

\section{Methods}

The included glaucoma suspect patients had CSLT followup measurements (at least 4 measurements) during their glaucoma routine checks at an ophthalmology institute. OD morphology with HRT III (Heidelberg Engineering GmbH, Heidelberg, Germany; software version 1.7) was measured without pupil dilation. In brief, a 3-dimensional topographic image consisting of $384 \times 384 \times 16$ up to $384 \times 384 \times 64$ voxels was constructed from multiple focal planes axially along the optic nerve head. To visualize the disc borders, the HRT images were inspected using the 3D image display. To define the contour line, six or more points were positioned at the inner margin of the scleral ring. This was done by one trained technician. Once the contour line was drawn, the software automatically calculated all the optic disc parameters. The reference plane is defined at $50 \mu \mathrm{m}$ posterior to the mean retinal height between 350 and 356 degrees along the contour line. The area above the reference is defined as the rim and below as the cup. The standard protocol and the extended parameter table were then exported for statistical analysis.

OD change during the CSLT follow-up was determined with strict, moderate, and liberal criteria of the TCA [25]. The TCA takes into account the size and depth of the largest contiguous area or cluster of significant change within the optic disc border. According to that, patients were divided into a stable and progressive glaucoma suspect group.

On the study day, an automated visual field testing was performed with the Humphrey field analyzer II (program $30-2$ ). Visual field eligibility criteria were less than $33 \%$ false-positive responses, less than $33 \%$ false-negative responses, and less than 33\% fixation losses. Subjects showing either pathologic glaucoma hemifield test or a cluster of 3 points in pattern deviation plot significant at $0.5 \%$ not located at the border of the visual field, were excluded. As well, Cirrus ${ }^{\circledR}$-SD-OCT (Carl Zeiss Meditec, Dublin, CA, USA) measurements centered in the macula (Macular Cube $200 \times 200$ ) and centered on OD were performed by one technician, using the optic disc cube $200 \times 200$. With this program, a data cube of $6-\mathrm{mm}^{2}$, which acquires as a series of 200 horizontal scan lines, each composed of 200 A-scans was recorded. Measurements of the OD parameters were automatically generated by a Carl Zeiss Meditec OD analysis algorithm developed for Cirrus ${ }^{\circledR}$-SD-OCT (software version 10.0.0.14618) without the interaction of the technician [27]. The RNFL was measured at a 3.4-mm-diameter circle around the OD. For both parameters, the software calculates the average, superior, nasal, inferior, and temporal quadrant values. 
Model compensation (MC) as well as age compensation (AC) was applied to the global, quadrants, and clock hour sectors of these RNFL thickness values.

\section{Statistical analysis}

Statistical analysis was performed using the SPSS $®$ software package (Version 26.0.0, SPSS Inc., USA) and R Version (3.6.0) [28]. Multivariate model compensation (MC), (for details see our previous manuscript) [24] as well as age compensation (AC) of RNFL (RNFLMC vs. RNFLAC) was applied to the global, quadrants, and clock hour sectors (CHR) of the peripapillary RNFL thickness values, for both patient groups, stable and progressive. Parameters included in the multivariate model were age, spherical refractive error, retinal vessel density, optic disc area, orientation, and ratio (quotient between major and minor axis), as well as disc-fovea distance and angle. These parameters are known to correlate with peripapillary RNFL distribution. To compensate for the effect of the above eight parameters on RNFL thickness, we used the multivariate model to calculate for each subject a model-compensated RNFL thickness (RNFLMC). For age compensation, we used the data of the instrument's normative database. Differences between groups regarding RNFL (RNFLMC and RNFLAC) and age were assessed using the Mann-Whitney $U$ tests and Student's $t$ test. $P$ values are provided as an explorative tool and were not corrected for multiple testing. $P$ values smaller than 0.05 indicate the difference between groups.

The diagnostic performance of RNFLMC vs RNFLAC vs originally measured RNFL was tested with the area under the receiver operating characteristic (AUROC) and

Table 1 Demographic data. Patient characteristics, $n=42$ glaucoma suspects. $S D$ standard deviation, $I O P$ intraocular pressure, $M D$ mean deviation, $C / D$ cup/disc ratio

\begin{tabular}{ll}
\hline & $n=42$ glaucoma suspects \\
\hline Female:male & $23: 19$ \\
Mean age \pm SD in years & $62.0 \pm 11.4$ \\
Mean \pm SD of total CSLT follow-up in & $104.4 \pm 39.2$ \\
$\quad$ months & \\
Mean number \pm SD of CSLT examinations & $9 \pm 3$ \\
IOP on the study day (mean \pm SD) & $16 \pm 3.57$ \\
$\quad$ (mmHg) & $-0.1 \pm 1.25$ \\
Visual field MD (mean \pm SD) (dB) & $0.7 \pm 0.07$ \\
C/D ratio (mean \pm SD) & $-1.04 \pm 2.02$ \\
Refractive error, spheric equivalent & \\
$\quad$ (mean \pm SD) (diopters) & 10 \\
Number of patients with glaucoma therapy &
\end{tabular}

compared between methods using DeLong's tests computed with the R package pROC [29].

\section{Results}

From the 54 glaucoma suspects screened, 12 had to be excluded due to insufficient image quality and/or retinal pathologies (for example vitreomacular traction) or visual field defects. A total of 42 glaucoma suspects with or without prior progressive OD change in a series of at least 4 CSLT (HRT®III measurements) were included. The mean follow-up time of measurements with HRT was 104.4 months. For statistical analysis, the last 3 measurements next to the study day were used. Subjects' baseline demographics are provided in Table 1.

OD change during the CSLT follow-up was determined with strict, moderate, and liberal criteria of the TCA. According to that, patients were divided into a stable and a progressive patient group for each of the three criteria. The number of glaucoma suspects with prior progressive optic disc change during CSLT follow-up was 38, 29, and 16 for liberal, moderate, and strict criteria of the TCA, respectively. The number of stable glaucoma suspects was 4 for the liberal, 13 for the moderate, and 26 for the strict criterion.

The application of the strict criterion of TCA leads to fewer numbers of progressive glaucoma suspect patients due to its conservative definition of requiring a significant cluster of $\geq 2 \%$ of the disc area and a depth change of $\geq 100 \mu \mathrm{m}$. In contrast, the liberal criterion required a significant cluster of $\geq 0.5 \%$ of the disc area and a depth change of $\geq 20 \mu \mathrm{m}$ and the moderate criterion a significant cluster of $\geq 1 \%$ of the disc area, and a depth change of $\geq 50 \mu \mathrm{m}$.

MC as well as AC of RNFL was applied to the global peripapillary RNFL thickness values measured with OCT, as well as to the quadrants thereof. For each instance, the differences in RNFL thickness and also in age between the two glaucoma suspect groups (stable versus progressive) were assessed with $t$-tests (Table 2).

Glaucoma suspect patients without prior progressive OD change during the CSLT follow-up (= stable) were significantly younger and had thicker RNFL thickness values in most areas and for all progression criteria. The only exception was the temporal quadrant for the moderate and strict criteria for originally measured RNFL as well as RNFLMC and RNFLAC, where the stable patients had thinner RNFL values, although generally not statistically significant. For the liberal criterion, stable glaucoma suspects (this were only 4 patients) had statistically significant larger RNFL thickness values globally and in the superior and inferior quadrant, originally measured but also after applying MC 
Table 2 Median values of peripapillary retinal nerve fiber layer thickness values (RNFL) in $\mu \mathrm{m}$ (and interquartile range) using original, model compensated (MC), and age compensated (AC) values for the two groups of glaucoma suspect patients, with (= progressive) or without (=stable) prior progressive optic disc change during the CSLT follow-up

\begin{tabular}{|c|c|c|c|}
\hline TCA & Progressive & Stable & $P$ values \\
\hline Liberal criteria $(n)$ & 38 & 4 & \\
\hline Age & $62.0( \pm 11.7)$ & $58.8( \pm 6.1)$ & 0.561 \\
\hline RNFL global & $85.5(80.5-91.4)$ & $\mathbf{9 8 . 5}(92.6-101.8)$ & $0.008 *$ \\
\hline Temporal quadrant & $62.0(56.3-70.1)$ & $62.5(54.3-70.1)$ & 0.932 \\
\hline Superior quadrant & $104.6(92.7-115.6)$ & $132.8(116.8-144.6)$ & $0.007 *$ \\
\hline Nasal quadrant & $67,7(64.4-78.5)$ & $68.7(64.0-79.4)$ & 0.830 \\
\hline Inferior quadrant & $106.7(96.2-116.2)$ & $\mathbf{1 2 6 . 0}(116.5-135.9)$ & $0.015^{*}$ \\
\hline RNFL global_MC & $85.7(80.9-88.7)$ & $97.0(94.0-99.0)$ & $0.004 *$ \\
\hline Temporal quadrant_MC & $61.5(56.2-66.6)$ & 63.6 (55.4-75.3) & 0.607 \\
\hline Superior quadrant_MC & $103.6(93.5-115.5)$ & $121.4(118.1-134.7)$ & $0.004 *$ \\
\hline Nasal quadrant_MC & $70.4(65.0-74.6)$ & $73.1(68.8-76.2)$ & 0.346 \\
\hline Inferior quadrant_MC & $105.4(96.3-113.9)$ & $126.6(118.8-135.0)$ & $0.005 *$ \\
\hline RNFL global_AC & $87.7(84.0-94.5)$ & $100.7(94.2-105.1)$ & $0.016^{*}$ \\
\hline Temporal quadrant_AC & $64.3(57.2-71.4)$ & $63.8(55.1-71.7)$ & 1.000 \\
\hline Superior quadrant_AC & $108.3(97.0-118.7)$ & $135.7(118.4-149.2)$ & $0.008 *$ \\
\hline Nasal quadrant_AC & $70.1(65.6-79.5)$ & $70.0(65.6-80.2)$ & 0.898 \\
\hline Inferior quadrant_AC & $109.0(101.9-122.1)$ & $\mathbf{1 3 0 . 0}(120.5-139.5)$ & $0.015^{*}$ \\
\hline Moderate criteria $(n)$ & 29 & 13 & \\
\hline Age & $65.0( \pm 10.8)$ & $55.3( \pm 8.7)$ & $0.003^{*}$ \\
\hline RNFL global & $85.4(80.3-90.1)$ & $91.5(84.5-96.7)$ & $0.062 *$ \\
\hline Temporal quadrant & $\mathbf{6 5 . 8}(56.6-70.6)$ & $58.3(54.4-65.1)$ & 0.178 \\
\hline Superior quadrant & $103.1(91.9-113.2)$ & $115.4(105.8-124.7)$ & $0.027 *$ \\
\hline Nasal quadrant & $\mathbf{6 7 . 9}(64.7-75.8)$ & $66.8(63.5-81.5)$ & 0.881 \\
\hline Inferior quadrant & $105.4(95.5-115.1)$ & $114.1(105.5-127.9)$ & 0.055 \\
\hline RNFL global_MC & $84.0(79.8-88.2)$ & $89.4(85.7-96.0)$ & $0.025 *$ \\
\hline Temporal quadrant_MC & $\mathbf{6 3 . 6}(58.0-68.2)$ & $57.4(55.3-65.2)$ & 0.178 \\
\hline Superior quadrant_MC & $103.3(88.5-113.5)$ & $115.4(101.7-118.6)$ & $0.066^{*}$ \\
\hline Nasal quadrant_MC & $69.1(65.0-74.4)$ & $72.0(68.5-75.5)$ & 0.391 \\
\hline Inferior quadrant_MC & $104.9(93.8-113.1)$ & $114.3(103.6-126.6)$ & $0.031 *$ \\
\hline RNFL global_AC & $87.8(83.9-94.3)$ & $\mathbf{9 2 . 6}(85.2-99.2)$ & 0.334 \\
\hline Temporal quadrant_AC & $\mathbf{6 7 . 9}(58.8-72.2)$ & $58.8(55.3-66.3)$ & $0.084 *$ \\
\hline Superior quadrant_AC & $108.1(96.7-117.0)$ & $\mathbf{1 1 4 . 3}(106.1-127.3)$ & 0.131 \\
\hline Nasal quadrant_AC & $70.1(66.4-77.8)$ & $68.7(64.4-82.3)$ & 0.838 \\
\hline Inferior quadrant_AC & $109.2(100.8-122.1)$ & $\mathbf{1 1 8 . 3}(106.1-129.7)$ & 0.178 \\
\hline Strict criteria $(n)$ & 16 & 26 & \\
\hline Age & $66.6( \pm 10.1)$ & $58.7( \pm 11.1)$ & $0.02 *$ \\
\hline RNFL global & $86.0(76.5-88.9)$ & $87.3(83.1-95.3)$ & 0.133 \\
\hline Temporal quadrant & $67.3(56.9-72.3)$ & $59.8(55.3-67.0)$ & 0.120 \\
\hline Superior quadrant & $102.4(89.5-115.8)$ & $107.4(97.7-119.8)$ & 0.178 \\
\hline Nasal quadrant & $65.9(62.8-71.5)$ & $\mathbf{7 1 . 4}(65.0-80.2)$ & $0.062 *$ \\
\hline Inferior quadrant & $101.6(93.3-111.0)$ & $113.1(103.4-124.1)$ & $0.024 *$ \\
\hline RNFL global_MC & $85.3(78.8-88.2)$ & $87.1(83.5-94.3)$ & $0.087 *$ \\
\hline Temporal quadrant_MC & $64.8(60.0-68.5)$ & $58.4(55.7-66.2)$ & 0.178 \\
\hline Superior quadrant_MC & $98.2(92.8-114.1)$ & $\mathbf{1 0 6 . 5}(99.3-117.3)$ & 0.204 \\
\hline Nasal quadrant_MC & $67.3(65.0-72.6)$ & $72.3(66.3-77.2)$ & $0.062 *$ \\
\hline Inferior quadrant_MC & $101.1(91.2-111.2)$ & $112.6(101.5-107.4)$ & $0.034 *$ \\
\hline RNFL global_AC & $86.9(81.7-93.9)$ & $88.1(85.4-98.0)$ & 0.254 \\
\hline Temporal quadrant_AC & $70.1(59.5-73.6)$ & $60.4(55.8-68.9)$ & $0.059^{*}$ \\
\hline Superior quadrant_AC & $108.5(94.1-120.5)$ & $\mathbf{1 1 0 . 3}(101.1-121.4)$ & 0.484 \\
\hline Nasal quadrant_AC & $67.9(65.0-74.2)$ & $\mathbf{7 2 . 4}(65.6-81.8)$ & 0.147 \\
\hline Inferior quadrant_AC & $105.8(99.4-116.0)$ & $\mathbf{1 1 5 . 6}(104.9-125.8)$ & $0.074 *$ \\
\hline
\end{tabular}

Group status was determined with liberal, moderate, and strict criteria of the topographic change analysis (TCA). Differences between groups were assessed using Student's $t$ test. $P$ values are provided as an explorative tool and were not corrected for multiple testing. Bold $=$ thicker RNFL values $(>1 \mu \mathrm{m}$, statistical significance not required), ${ }^{*} P$ values smaller than 0.1 indicate some difference between groups 
Table 3 Comparison of diagnostic performance of multivariate model compensation (MC) as well as age compensation (AC) of retinal nerve fiber layer (RNFL) thickness parameters using area under the receiver operating characteristics (AUROC) for liberal, moderate, and strict criteria of the topographic change analysis in glaucoma suspect patients

\begin{tabular}{llll}
\hline & AC AUROC & MC AUROC & $P$ values \\
\hline Liberal criteria & & & \\
RNFL global & 0.868 & $\mathbf{0 . 9 4 7}$ & 0.107 \\
Temporal quadrant & 0.500 & 0.579 & 0.805 \\
Superior quadrant & $\mathbf{0 . 9 0 8}$ & $\mathbf{0 . 9 4 7}$ & 0.460 \\
Nasal quadrant & 0.520 & 0.645 & 0.450 \\
Inferior quadrant & 0.875 & 0.928 & 0.234 \\
Moderate criteria & & & \\
RNFL global & 0.594 & $\mathbf{0 . 7 1 9}$ & $0.028^{*}$ \\
Temporal quadrant & 0.332 & 0.371 & 0.436 \\
Superior quadrant & $\mathbf{0 . 6 4 7}$ & 0.679 & 0.449 \\
Nasal quadrant & 0.480 & 0.584 & 0.594 \\
Inferior quadrant & 0.631 & 0.711 & $0.024 *$ \\
Strict criteria & & & \\
RNFL global & 0.606 & 0.659 & 0.354 \\
Temporal quadrant & 0.325 & 0.375 & 0.277 \\
Superior quadrant & 0.565 & 0.618 & 0.398 \\
Nasal quadrant & 0.635 & 0.673 & 0.656 \\
Inferior quadrant & $\mathbf{0 . 6 6 6}$ & $\mathbf{0 . 6 9 7}$ & 0.401 \\
\hline
\end{tabular}

Bold $=$ best performing parameters in terms of AUROC regarding MC or AC (cutoff $\geq 0.01$ ) $* P$ values smaller than 0.05 indicate the difference between groups

and AC. For the strict criterion, progressive glaucoma suspects had statistically significant thinner RNFL thickness values in the inferior quadrant, again for all three methods, originally measured and after applying MC and AC.

Diagnostic performance of RNFLMC vs RNFLAC was quantified with area under the receiver operating characteristic (AUROC) and compared between methods using DeLong's test (Table 3). Due to the significant age differences between the stable and the progressive groups, we do not report in detail on the AUROC as obtained by the originally measured RNFL.

For glaucoma suspects, in general, liberal criteria of HRT progression performed better concerning AUROC than moderate or strict criteria (Table 3), regardless of the compensation method. But the interpretation that the liberal criterion is better regarding diagnostic separation is speculative, as a small sample size in the stable group $(n=4)$ impedes significant results.

MC AUROC of global RNFL and of the inferior quadrant performed statistically significantly better than AC AUROC, for the moderate criterion (see Fig. 1a-c for the AUROCs of the RNFL global).
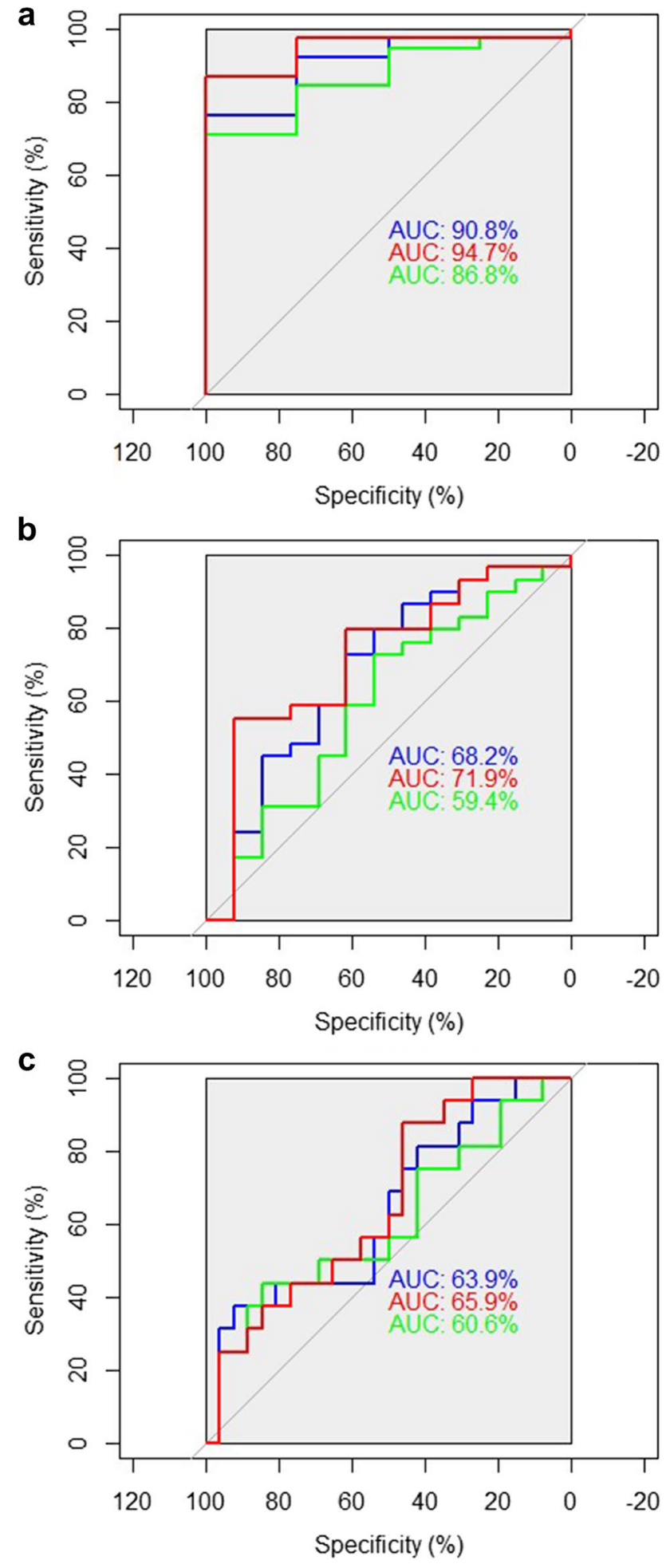

Fig. 1 a AUC global RNFL thickness parameters, liberal criteria. Blue, RNFL_Average (original); red, RNFL_Average (model compensated, MC_AUROC); green, RNFL_Average (age compensated, AC_AUROC). b AUC global RNFL thickness parameters, moderate criteria. Blue, RNFL_Average (original); red, RNFL_Average (model compensated, MC_AUROC); green, RNFL_Average (age compensated, AC_AUROC). c AUC global RNFL thickness parameters, strict criteria. Blue, RNFL_ Average (original); red, RNFL_Average (model compensated, $\mathrm{MC}_{-}$ AUROC); green, RNFL_Average (age compensated, AC_AUROC) 
In general, original RNFL and MC AUROC performed better than AC AUROC for the moderate progression criterion. For the strict criterion, these differences were less pronounced.

AUROC for originally measured RNFL performed statistically significantly better than AC AUROC for the superior, nasal, and inferior quadrant for moderate and strict criteria and for RNFL global for the moderate criterion. But taking into account the age distribution for stable and progredient glaucoma suspects, it became obvious that stable glaucoma suspects were statistically significantly younger than progredient ones by 10 or 8 years for the moderate and the strict criteria. This leads to bias in favor of the originally measured RNFL. In Fig. 1, the AUROCs for global RNFL thickness parameters are shown and the respective curves for the originally measured RNFL do not take into account the age differences, while both the $\mathrm{AC}$ and the $\mathrm{MC}$ do correct for age differences.

\section{Discussion}

In this paper, we use our prior published multivariate model that facilitates a clinically meaningful reduction of the intersubject variance of peripapillary RNFL parameters, by compensating for variation of parameters correlating with RNFL thickness distribution. This model includes physiological parameters, automatically extracted from OCT fundus images such as retinal vessel density, OD shape, fovea location, and additionally age and refractive error [23, 24].

This model (MC) was applied on RNFL parameters measured with Cirrus OCT in patients with suspicion of glaucoma. This patient collective was divided into 2 groups, a group with or without prior progressive OD change in a series of at least 4 HRT®III measurements. Change during the CSLT follow-up was determined with strict, moderate, and liberal criteria of the TCA. MC as well age compensation (AC) of RNFL was applied to the global, quadrants, and clock hour sectors of RNFL thickness values. It is to be expected that the MC leads to a better diagnostic separation. For glaucoma suspect patients, trends are seen in the present paper of MC performing better in regards of AUROC in comparison to $\mathrm{AC}$, for all progression criteria, with statistically significant differences for global RNFL and the inferior quadrant (a clinically relevant region for glaucoma damage) for the moderate criterion. The lack of significance for all other parameters might be on the one hand due to the small sample size, especially for stable patients according to the liberal criteria. On the other hand, the limitation of HRT as a method to detect glaucoma progression (OD change over time) might have had an influence.

There is no consensus regarding the ability of HRT to detect progression among the prior literature. One study showed that TCA parameters observed longitudinally can discriminate between progressing and healthy eyes [25]. However, another prospective study suggested that TCA progression criteria do not predict photographic evaluation or visual field progression [30]. Similarly, a retrospective study demonstrated that the stereometric parameters of the HRT did not have a high enough sensitivity and specificity to detect glaucomatous progression that was otherwise detected by serial stereoscopic OD photography evaluated by experienced masked observers [31]. There are certain factors that can influence measurement reliability. The HRT estimates the height location of surface pixels indirectly through the use of a reference plane, which is set at an arbitrarily determined depth below the outer limit of the RNFL in the papillomacular bundle. This reference plane may be subject to alteration due to progressing glaucomatous damage at this location. This in turn may affect changes in pixel height as assessed by TCA.

In the present paper, 10 patients were treated with eye pressure-lowering eye drops, only 1 patient needed therapy enhancement (from mono-therapy to a combination treatment), and no glaucoma surgery was performed during the HRT measurement period. An influence of treatment on the measurement results seems thus unlikely.

The better performance of the AUROCs for liberal criteria in glaucoma suspect patients when compared to the other two criteria might indicate that in our setting a large proportion of patients with progressive OD change according to liberal criteria truly have glaucoma whereas, for strict criteria, many of them are labeled as (false) negatives. On the other hand, only when progression was defined by the moderate criterion the inferior quadrant RNFL MC and global RNFL MC performed significantly better than AC.

In our data, AUROC for originally measured RNFL performed statistically significantly better than RNFL AC AUROC for the superior, nasal, and inferior quadrant for moderate and strict criteria. However, lack of age compensation introduces a relevant bias when using the originally measured RNFL values in our data, where progressive glaucoma suspects were older by 8 to 10 years compared to stable glaucoma suspects. Furthermore, it is the AC compensation method, which is commonly applied during OCT data evaluation in daily routine, whereas the originally measured RNFL values, without age correction or age-dependent normative values, are generally neither clinically nor scientifically used.

A sample bias might also have had an influence on the study results, as patients with newly developed visual field defects were excluded. Only patients with normal visual fields were recruited, but if they had reproducible scotomas on the study day, they were excluded from further study participation. This was the case in 10 glaucoma suspects, which might lead to an inclusion of only stable glaucoma suspects. For these 10 glaucoma suspects, we also executed the strict, moderate, 
and liberal criteria of the TCA. For the liberal criterion, all of these glaucoma suspects were labeled as progressive (10 out of 10). Regarding the moderate criterion, 6 out 10 , and, for the strict criteria, 4 out of 10 , glaucoma suspects were classified with progressive optic disc change. The interpretation of this analysis could be that with strict criteria of the HRT-TCA real glaucoma cases might be missed; and on the other hand, for the liberal criterion, healthy subjects might be labeled as glaucoma. CSLT is able to detect optic disc change over time, but its clinical impact regarding real glaucoma disease progression should be interpreted with caution.

In conclusion, we found some evidence that the reduction of interindividual variability of RNFL measurements through using a multivariate compensation method might improve the detection of glaucoma among glaucoma suspect patients. Further studies are warranted to validate the present results.

Funding Open access funding provided by Medical University of Vienna.

Data availability (data transparency) On demand.

Code availability (software application or custom code) On demand.

\section{Declarations}

Ethics approval The study was approved by the local ethics committee board of the medical university of Vienna, Austria. All procedures performed in studies involving human participants were in accordance with the ethical standards and with the 1964 Helsinki declaration and its later amendments or comparable ethical standards.

Informed consent Informed consent was obtained from all individual participants included in the study.

Consent for publication All authors agree with the publication.

Conflict of interest The authors declare no competing interests.

Open Access This article is licensed under a Creative Commons Attribution 4.0 International License, which permits use, sharing, adaptation, distribution and reproduction in any medium or format, as long as you give appropriate credit to the original author(s) and the source, provide a link to the Creative Commons licence, and indicate if changes were made. The images or other third party material in this article are included in the article's Creative Commons licence, unless indicated otherwise in a credit line to the material. If material is not included in the article's Creative Commons licence and your intended use is not permitted by statutory regulation or exceeds the permitted use, you will need to obtain permission directly from the copyright holder. To view a copy of this licence, visit http://creativecommons.org/licenses/by/4.0/.

\section{References}

1. Kerrigan-Baumrind LA, Quigley HA, Pease ME et al (2000) Number of ganglion cells in glaucoma eyes compared with threshold visual field tests in the same persons. Invest Ophthalmol Vis Sci 41:741-748

2. Townsend KA, Wollstein G, Schuman JS (2009) Imaging of the retinal nerve fibre layer for glaucoma. Br J Ophthalmol 93:139-143

3. Carpineto P, Ciancaglini M, Zuppardi E et al (2003) Reliability of nerve fiber layer thickness measurements using optical coherence tomography in normal and glaucomatous eyes. Ophthalmology 110:190-219

4. Blumenthal EZ, Frenkel S (2005) Inter-device reproducibility of the scanning laser polarimeter with variable cornea compensation. Eye 19:308-311

5. Ghadiali Q, Hood DC, Lee C et al (2008) An analysis of normal variations in retinal nerve fiber layer thickness profiles measured with optical coherence tomography. J Glaucoma 17:333-340

6. Alasil T, Wang KD, Keane PA et al (2013) Analysis of normal retinal nerve fiber layer thickness by age, sex, and race using spectral domain optical coherence tomography. J Glaucoma 22:532-541

7. Budenz DL, Anderson DR, Varma R et al (2007) Determinants of normal retinal nerve fiber layer thickness measured by Stratus OCT. Ophthalmology 114:1046-1052

8. Celebi AR, Mirza GE (2013) Age-related change in retinal nerve fiber layer thickness measured with spectral domain optical coherence tomography. Invest Ophthalmol Vis Sci 54:8095-8103

9. Girkin CA, McGwin G Jr, Sinai MJ et al (2011) Variation in optic nerve and macular structure with age and race with spectral-domain optical coherence tomography. Ophthalmology 118:2403-2408

10. Lim LS, Cheung CY, Lin X et al (2011) Influence of refractive error and axial length on retinal vessel geometric characteristics. Invest Ophthalmol Vis Sci 52:669-678

11. Kang SH, Hong SW, Im SK et al (2010) Effect of myopia on the thickness of the retinal nerve fiber layer measured by Cirrus HD optical coherence tomography. Invest Ophthalmol Vis Sci 51:4075-4083

12. Valverde-Megias A, Martinez-de-la-Casa JM, Serrador-Garcia $M$ et al (2013) Clinical relevance of foveal location on retinal nerve fiber layer thickness using the new FoDi software in spectralis optical coherence tomography. Invest Ophthalmol Vis Sci 54:5771-5776

13. Hong SW, Ahn MD, Kang SH et al (2010) Analysis of peripapillary retinal nerve fiber distribution in normal young adults. Invest Ophthalmol Vis Sci 51:3515-3523

14. Choi JA, Kim JS, Park HY et al (2014) The foveal position relative to the optic disc and the retinal nerve fiber layer thickness profile in myopia. Invest Ophthalmol Vis Sci 55:1419-1426

15. Savini G, Zanini M, Carelli V et al (2005) Correlation between retinal nerve fibre layer thickness and optic nerve head size: an optical coherence tomography study. Br J Ophthalmol 89:489-492

16. Huang D, Chopra V, Lu ATH et al (2012) Does optic nerve head size variation affect circumpapillary retinal nerve fiber layer thickness measurement by optical coherence tomography? Invest Ophthalmol Vis Sci 53:4990-4997

17. Knight OJ, Girkin CA, Budenz DL et al (2012) Effect of race, age, and axial length on optic nerve head parameters and retinal nerve fiber layer thickness measured by Cirrus HD-OCT. Arch Ophthalmol 130:312-318

18. Nagai-Kusuhara A, Nakamura M, Fujioka M et al (2008) Association of retinal nerve fibre layer thickness measured by confocal scanning laser ophthalmoscopy and optical coherence tomography with disc size and axial length. Br J Ophthalmol 92:186-190

19. Yamashita T, Asaoka R, Tanaka M et al (2013) Relationship between position of peak retinal nerve fiber layer thickness and retinal arteries on sectoral retinal nerve fiber layer thickness. Invest Ophthalmol Vis Sci 54:5481-5488 
20. Hood DC, Fortune B, Arthur SN et al (2008) Blood vessel contributions to retinal nerve fiber layer thickness profiles measured with optical coherence tomography. J Glaucoma 17:519-528

21. Hood DC, Salant JA, Arthur SN et al (2010) The location of the inferior and superior temporal blood vessels and interindividual variability of the retinal nerve fiber layer thickness. J Glaucoma 19:158-166

22. Resch H, Brela B, Resch-Wolfslehner C et al (2011) Position of retinal blood vessels correlates with retinal nerve fibre layer thickness profiles as measured with GDx VCC and ECC. Br J Ophthalmol 95:680-684

23. Pereira I, Weber S, Holzer S et al (2014) Correlation between retinal vessel density profile and circumpapillary RNFL thickness measured with Fourier-domain optical coherence tomography. Br J Ophthalmol 98:538-543

24. Pereira I, Resch H, Schwarzhans F et al (2015) Multivariate model of the intersubject variability of the retinal nerve fiber layer thickness in healthy subjects. Invest Ophthalmol Vis Sci 56:5290-5298

25. Chauhan BC, Hutchison DH, Artes PH et al (2009) Optic disc progression in glaucoma: comparison of confocal scanning laser tomography to optic disc photographs in a prospective study. Invest Ophthalmol Vis Sci 50:1682-1691

26. Balasubramanian M, Bowd C, Vizzeri G et al (2009) Effect of image quality on tissue thickness measurements obtained with spectral domain-optical coherence tomography. Opt Express $17: 4019-4036$
27. Mwanza JC, Oakley JD, Budenz DL et al (2011) Cirrus Optical CoherenceTomography Normative Database Study Group. Ability of cirrus HD-OCT optic nerve head parameters to discriminate normal from glaucomatous eyes. Ophthalmology 118:241-248

28. R Core Team (2019) R: A language and environment for statistical computing. R Foundation for Statistical Computing, Vienna, Austria. URL https://www.R-project.org/.17.05.2021. Accessed 17 May 2021

29. Robin X, Turck N, Hainard A, Tiberti N, Lisacek F, Sanchez JC et al (2011) pROC: an open-source package for R and S+ to analyze and compare ROC curves. BMC Bioinformatics 12:77

30. Kourkoutas D, Buys YM, Flanagan JG et al (2014) Clinical significance of optic disc progression bytopographic change analysis maps in glaucoma: an 8-year follow-up study. J Ophthalmol 2014:987389. https://doi.org/10.1155/2014/987389

31. Saarela V, Falck A, Airaksinen PJ, Tuulonen A (2010) The sensitivity and specificity of Heidelberg Retina Tomograph parameters to glaucomatous progression in disc photographs. Br J Ophthalmol 94:68-73

Publisher's note Springer Nature remains neutral with regard to jurisdictional claims in published maps and institutional affiliations. 